Research Paper

\title{
Regulative Effect of Nampt on Tumor Progression and Cell Viability in Human Colorectal Cancer
}

\author{
Xiaoqun Lv1, Lingyun Zhang2, 3, Yanyan Zhu', Harun M. Said ${ }^{5}$, Jimin Shi², Guoxiong $\mathrm{Xu}^{2}$, 3, \\ 1. Department of Pharmacy, Jinshan Hospital, Fudan University, Shanghai 201508, China; \\ 2. Center Laboratory, Jinshan Hospital, Fudan University, Shanghai 201508, China; \\ 3. Department of Oncology, Shanghai Medical College, Fudan University, Shanghai 200032, China; \\ 4. Department of General Surgery, Jinshan Hospital, Fudan University, Shanghai 201508, China; \\ 5. Department of Radiation Oncology, University of Würzburg, D-97080 Würzburg, Germany
}

$\triangle$ Corresponding author: Guoxiong Xu, MD, PhD. Center Laboratory, Jinshan Hospital, Fudan University, 1508 Longhang Road, Shanghai 201508, China. Tel: +86-21-34189990. Fax: +86-21-57039502. Email: guoxiong.xu@fudan.edu.cn

() 2015 Ivyspring International Publisher. Reproduction is permitted for personal, noncommercial use, provided that the article is in whole, unmodified, and properly cited. See http://ivyspring.com/terms for terms and conditions.

Received: 2015.04.07; Accepted: 2015.06.16; Published: 2015.07.16

\begin{abstract}
Colorectal cancer (CRC) is the third most common cancer disease. Here we examined Nampt expression in patients with CRC and the effect of Nampt on cell viability in CRC cells. Nampt protein was overexpressed in colorectal adenoma as well as colorectal carcinoma. The immunoreactive staining of Nampt was negative in the adjacent normal colorectal tissue, weak in colorectal adenoma, and strong in colorectal carcinoma, which may represent tumor progression. Further evaluation of clinical data showed that Nampt expression was not correlated with the clinicopathological characteristics of CRC. Additionally, our in vitro studies demonstrated that Nampt promotes CRC cell viability, whereas the Nampt inhibitor FK866 suppressed CRC cell viability, which was in concordance with the previous studies in other cancer cells. Treatment with Nampt-siRNA reduced the Nampt protein expression resulting in the inhibition of the cell viability of HCT1 16 and Caco2. Thus, the involvement of Nampt in cell growth indicates that Nampt may play an important role in colorectal tumorigenesis. As a consequence, our results suggest that Nampt may be considered as a progression marker of colorectal tumor and a potentially therapeutic target for the treatment of CRC.
\end{abstract}

Key words: Nampt; visfatin; PBEF; tumor biomarker; adenocarcinoma; cell proliferation

\section{Introduction}

Colorectal cancer (CRC) is the third most common cancer and the third leading cause of cancer death expected to occur in both men and women in the United States in 2014 [1] and accounted for almost $10 \%$ of global cancer incidence burden in 2012 [2]. Initially, it is developed as a benign precursor lesion (adenoma), which can progress to an invasive lesion (adenocarcinoma) with the capacity to metastasize (metastatic adenocarcinoma). The 5-year survival rate of this disease is approximately $90 \%$ for patients with localized disease and approximately $66 \%$ for patients with regional disease, as determined at diagnosis [3]. Obesity as one of risk factors has been reported to be associated with colorectal adenomas [4]. Recent epidemiological and experimental evidence indicates that obesity is related to the metabolic abnormalities that are associated with the development of certain types of epithelial malignancies, including CRC [5]. Pathological and genetic observations demonstrated that colorectal adenoma precedes the majority of colorectal carcinoma with the possibility of undergoing a malignant transformation into carcinoma. Although numerous genetic alterations have been known to be involved in the development of CRC, the molecular mechanisms underlying this colorectal carcinogenesis and the molecular alterations that are responsible for 
the regulation of this disease are still not clear [6]. Thus, understanding the mechanisms involved in the initiation and progression of CRC is a pivotal step for the generation of optimal and more effective therapeutic approaches.

Nicotinamide

phosphoribosyltransferase (Nampt), also known in the literature as visfatin or pre-B cell enhancing factor (PBEF) which was isolated from peripheral blood lymphocytes and described as a secreted growth factor for early B cell proliferation [7], possesses many important functions for cellular patho-physiology being an insulin-mimetic adipokine which is highly expressed and secreted by visceral adipose tissue, associated with obesity [8]. Furthermore, Nampt is the rate-limiting enzyme that converts nicotinamide into $\mathrm{NAD}^{+}$which is essential for cellular metabolism, energy production, DNA repair, and survival $[9,10]$. It is well known that cancer cells have a higher basal turnover of NAD and also display higher energy requirements compared with non-malignant cells [11]. Increasing evidence demonstrated that Nampt acts as a multifunctional enzyme and is important for the metabolism and immune response as well as cancer disease development [12].

The overexpression of Nampt has been found in human malignant tumors of different types, including colorectal carcinoma, gastric cancer, prostate cancer, ovarian cancer, breast cancer, thyroid carcinoma, endometrial cancer, myeloma, melanoma, astrocytomas/glioblastoma and other carcinomas [13-22]. Nampt was first reported to be overexpressed in colorectal cancer using suppression subtractive hybridization [15]. Additionally, plasma Nampt levels in patients with advanced and early cancer were higher than in controls [23, 24]. However, the expression of Nampt in both of human colorectal adenoma and carcinoma tissues was not explored yet. Furthermore, whether Nampt expression is associated with clinicopathological characteristics in colorectal carcinoma tissue is unknown.

FK866, also known as APO866, is a potent and specific small molecule inhibitor of Nampt, and suppresses the cancer cell growth in addition to the induction of apoptosis by NAD depletion [25]. Thus, FK866 appears to be an ideal tool molecule for examining the physiological function of Nampt in the cancer cell. The present study was undertaken to explore the expression of Nampt in human colorectal adenomas and carcinomas as well as to investigate whether Nampt expression is correlated with clinicopathological characteristics in colorectal carcinomas. Furthermore, we evaluated the effect of Nampt, its inhibitor FK866 and Nampt-siRNA on cell proliferation in CRC cell lines HT29, Caco2, and HCT116, respectively.

\section{Materials and methods}

\section{Colorectal tissue specimen collection from human patients}

Conducting the study on human tissue specimens was approved by the Ethics Committee of Jinshan Hospital, Fudan University at Shanghai, China. A total of 117 paraffin-embedded samples constituted by 35 adjacent normal colorectal tissues, 25 colorectal adenomas and 57 colorectal carcinomas were subjected to histo-pathological and clinical diagnosis at Jinshan Hospital from 2012 to 2013. None of the patients had received radiotherapy or chemotherapy before surgery.

The $10 \%$ formalin-fixed paraffin-embedded colorectal tissue specimens were collected and pathologically diagnosed in the Department of Pathology. Four micrometer thick sections of these specimens were stained by hematoxylin and eosin (H\&E) to confirm the histological characteristics as well as the final diagnosis with both the tumor stage and grade.

\section{Immunohistochemical staining and analysis}

Four micrometer tissue sections were baked at $60^{\circ} \mathrm{C}$ for $2 \mathrm{~h}$, followed by paraffin removal in xylene and rehydrating them with a series of graded alcohols. Subsequently, the sections were immersed in EDTA antigen retrieval solution (Maixin Bio, Fuzhou, Fujian, China) and heated in boiling water bath at $100^{\circ} \mathrm{C}$ for $20 \mathrm{~min}$. Endogenous peroxidase activity was blocked by $3 \%$ hydrogen peroxide at room temperature for $15 \mathrm{~min}$. Non-specific signal was blocked by $10 \%$ normal goat serum (Maixin Bio) at room temperature for $40 \mathrm{~min}$, and then the sections were incubated with a monoclonal rabbit anti-Nampt antibody (1:250 dilution, Abcam, Cambridge, UK) at $4^{\circ} \mathrm{C}$ overnight, followed by the incubation with biotinylated anti-rabbit secondary antibody (Maixin Bio) at room temperature for $1 \mathrm{~h}$. The first antibody was omitted for a negative control. After washing, the signal was detected using a DAB Kit (Maixin Bio). Finally, the sections were counterstained with hematoxylin and examined with image record under a light microscope (Olympus, Tokyo, Japan).

Scoring of Nampt immunoreactive staining was performed independently by two examiners who had no prior knowledge of patient's clinical status. The sections were evaluated at two magnifications (x100 and $x 400)$. The proportion of cells exhibiting Nampt expression was scored by the extent of immunoreactive staining and was assigned to one of the following categories as described previously [26]. Briefly, the percentage of positive cells was scored as given in the following: 0 , no positive cells; $1, \leq 25 \%$ positive cells; 2 , $26-50 \%$ positive cells; $3,51-75 \%$ positive cells; $4,>75 \%$ 
positive cells. The intensity of staining was scored as: 0 , no staining; 1 , weak staining; 2 , moderate staining; 3 , strong staining. The final staining index (SI) was developed based on the sum score of the positive staining and intensity. The SI was then clustered into four groups: ' 0 ', $\leq 2$ sum points; ' 1 ', 3-4 sum points; ' 2 ', $5-6$ sum points; ' 3 ', 7 sum points. Finally, the cases with grades equal to 0 and 1 were defined as Nampt negative and those with grades equal to 2 and 3 were defined as Nampt positive.

\section{Cell culture and transfection with siRNA,}

Human-derived CRC cell lines HT29, Caco-2, and HCT116 were obtained from the Institute of Cell Biology, Chinese Academy of Sciences (Shanghai, China). The cells were maintained in RPMI 1640 medium (HyClone, Thermo Fisher Scientific Inc., Beijing, China), MEM (HyClone), and McCoy's 5A medium (Sigma-Aldrich, Saint Louis, MO, USA), respectively, supplemented with $10 \%$ fetal bovine serum (HyClone) at $37^{\circ} \mathrm{C}$ in a humidified $5 \% \mathrm{CO}_{2}$ atmosphere.

Human Nampt-siRNA was a pool of 3 target-specific siRNAs designed to knock down gene expression (sc-45843, Santa Cruz Biotechnology, Inc., Dallas, TX, USA). Non-targeting siRNA (sc-37007, Santa Cruz) was used as a negative control. The cells were plated in 96-or 6-well plates for $24 \mathrm{~h}$ and then transfected with siRNA using the transfection reagent Lipofectamine RNAiMAX (Invitrogen Life Technologies, Carlsbad, CA, USA) according to the manufacturer's instructions. After transfection, the cells were subjected to further Cell Counting Kit-8 (CCK-8) assay or to protein extraction for Western blotting.

\section{Treatment of Nampt orFK866}

The recombinant human Nampt (cat \# SRP4908) and its inhibitor FK866 (cat \# F8557) were obtained from Sigma. HT29, Caco2, and HCT116 cells were seeded in 96-well culture plates at 4000 cells/well and allowed to attach for $24 \mathrm{~h}$ in an incubator. The complete medium was then replaced with serum-free medium for $16 \mathrm{~h}$ to allow for cell cycle synchronization. The cells were then either incubated with recombinant human Nampt at the concentration of 0 , 25,50 , and $100 \mathrm{ng} / \mathrm{ml}$ or FK866 at the concentration of 0,50 and $100 \mathrm{nM}$ for 24,48 , and $72 \mathrm{~h}$, respectively.

\section{Cell viability measurement}

The cell viability was determined using CCK-8 kit (Dojindo Laboratories, Kumamoto, Japan). Briefly, after incubation with Nampt or FK866 for 24, 48 or 72 h, $10 \mu \mathrm{l}$ of CCK- 8 reagent was added to each well, and the plates were further incubated in an incubator for 1 h. Subsequently, the absorbance at $490 \mathrm{~nm}$ was measured by a microplate reader (Bio-Rad Laboratories, Inc., Hercules, CA, USA). The cell viability (\%) was calculated as: (average OD of treated groups at 0 , 24,48 or $72 \mathrm{~h}$ /average OD of untreated groups at the same time point) $\times 100$.

\section{Western blot analysis}

The cells were lysed on ice with SDS lysis buffer (Beyotime, Haimen, Jiangsu, China) supplemented with 1\% PMSF (Beyotime) and 1\% protease inhibitor Cocktail (Sangon Biotech, Shanghai, China), followed by sonication. Besides, the fresh tumor tissues and their adjacent normal colorectal tissues from four patients with CRC were obtained by surgical resection and proteins were extracted with SDS lysis buffer. Protein samples were subjected to centrifugation, quantification and subsequently boiled prior to its analysis. Equal amounts of total protein $(30 \mu \mathrm{g})$ were separated on $10 \%$ SDS-PAGE and transferred to a PVDF membrane (Millipore, Billerica, MA, USA). After blocking with 5\% non-fat milk (Sangon Biotech) in Tris-buffered saline with Tween-20 (TBS-T) at room temperature for $2 \mathrm{~h}$, the membrane was incubated with a primary antibody at $4^{\circ} \mathrm{C}$ overnight followed by the incubation with an horseradish peroxidase-conjugated goat anti-rabbit IgG (1:10,000 dilution, Abcam) or anti-mouse IgG (1:10,000 dilution, Cell Signaling Technology, Inc., Danvers, MA, USA) at room temperature for $1 \mathrm{~h}$. The relative protein levels were calculated using GAPDH or $\beta$-actin as a loading control. The following primary antibodies were used: rabbit anti-Nampt (1:1000 dilution, Sig$\mathrm{ma})$, mouse anti-GAPDH (1:5000 dilution, Proteintech, Chicago, IL, USA), mouse anti- $\beta$-actin (1:2000 dilution, Proteintech). Signals were detected using Immobilon ${ }^{\mathrm{TM}}$ Western Chemiluminescent HRP Substrate (Millipore) and quantified using Tanon-4500 Gel Imaging System with GIS ID Analysis Software v4.1.5 (Tanon Science \& Technology Co., Ltd., Shanghai, China).

\section{Statistical analysis}

All statistical analyses were performed with SPSS Statistics 17.0 software (SPSS, Chicago, IL, USA). Based on the SI system, the categories on positivity and negativity were classified. Statistical evaluation was performed using $\chi^{2}$ test or Fisher's exact test to compare the positivity between two groups of the colorectal tissues and to analyze the correlation between Nampt expression and the clinicopathological characteristics. For comparison between the two scoring groups of immunostaining, the Mann-Whitney U test was applied. Significance levels for comparison of differences between experimental and control groups were analyzed by a Student's t-test. Results are presented as the mean \pm standard error of mean (SEM). At least 3 independent experi- 
ments were performed for each experimental series. Significant difference was considered at the value of $\mathrm{P}<0.05$.

\section{Results}

\section{Nampt as a progress marker for human colo- rectal adenoma and carcinoma}

By immunohistochemistry staining, the aberrant expression of Nampt was observed in human colorectal adenoma and adenocarcinoma, compared with the adjacent normal colorectal tissue that all showed negative staining. The highest degree of overexpression of Nampt was detected in colon and rectum adenocarcinomas (Fig. 1A and 1B). We found that Nampt was predominantly located in the cytoplasm with no defined membranous staining. Based on the SI system as indicated above, the level of Nampt expression in the tissues was classified into positive and negative categories. Compared with the adjacent normal colorectal tissue, the positive rate of Nampt expression was significantly higher in human colorectal adenoma $(P<0.05)$ and carcinoma $(P<0.001)$ (Table 1). Furthermore, the positive rate of Nampt expression in the malignant colorectal adenocarcinoma was higher than in the benign colorectal adenoma $(P<0.001)$ (Table 1$)$. The same conclusion was drawn from the statistical analysis of the immunoreactive scores of Nampt protein between the types of tissues examined (Table 2). Briefly, Nampt expression showed a remarkable increase from the adjacent normal tissue to colorectal carcinoma through adenoma $(P<0.001)$. The overexpression of Nampt in tumor tissues was further confirmed by Western blot. We found that there was about 1.5 times increase of Nampt expression in tumor tissues compared with matched adjacent normal colorectal tissues (Fig. 1C and 1D). These data suggest that Nampt may be a progression marker of the colorectal tumor. Nampt expression level alteration between the colorectal adenoma and carcinoma suggests that Nampt might be one of the inductive factors of carcinogenesis during the colorectal neoplastic transformation.

Table 1 Nampt expression in colorectal tissues

\begin{tabular}{lllll}
\hline Histology & $\mathrm{n}$ & \multicolumn{2}{c}{ Nampt expression } & P-value \\
\cline { 3 - 4 } & & Positive $\mathrm{n}(\%)$ & Negative $\mathrm{n}(\%)$ & $0.026^{\mathrm{a}}$ \\
Normal & 35 & $0(0)$ & $35(100)$ & $<0.001^{\mathrm{b}}$ \\
Adenoma & 25 & $4(16)$ & $21(84)$ & $<0.001^{\mathrm{c}}$ \\
Carcinoma & 57 & $35(61)$ & $22(39)$ & \\
\hline
\end{tabular}

Normal adjacent normal colorectal tissue, adenoma colorectal benign tumor, carcinoma colorectal malignant tumor, $n$ number of cases

a normal $v s$. adenoma, Fisher's exact test; ${ }^{b}$ adenoma $v$ s. carcinoma, $\chi^{2}$ test; ${ }^{c}$ normal vs. carcinoma, $\chi^{2}$ test
Table 2 Histological types and their relationship to Nampt expression

\begin{tabular}{|c|c|c|}
\hline Comparison & Z score & P-value \\
\hline Normal vs. adenoma & -6.318 & $<0.001$ \\
\hline Normal vs. carcinoma & -7.747 & $<0.001$ \\
\hline Carcinoma vs. adenoma & -4.156 & $<0.001$ \\
\hline \multicolumn{3}{|c|}{$\begin{array}{l}\text { Normal adjacent normal colorectal tissue; adenoma colorectal benign tumor; } \\
\text { noma colorectal malignant tumor }\end{array}$} \\
\hline \multicolumn{3}{|c|}{$\begin{array}{l}\text { The Mann-Whitney U test was used to analyze Nampt immunoreactive scor } \\
\text { between two types of tissues }\end{array}$} \\
\hline \multicolumn{3}{|c|}{$\begin{array}{l}\text { Correlation of Nampt expression with the } \\
\text { colorectal malignant tumor clinical and } \\
\text { pathological characteristics }\end{array}$} \\
\hline
\end{tabular}

For correlation between Nampt expression and the clinicopathological characteristics in CRC, either the Chi-square test or the Fisher's exact test was performed as indicated. All information listed in Table 3 was collected by reviewing medical charts and pathology records. By comparison of Nampt protein expression associated with gender and age, we found that Nampt expression was not significantly different between male and female as well as between younger ( $<60$ years) and older ( $\geq 60$ years) patients with CRC $(P>0.05)$. The positive rate of Nampt expression was $52.9 \%$ and $73.9 \%$ in colon and rectum, respectively, without significant difference between two regions $(P>0.05)$. Moreover, there was no relationship between Nampt expression and tumor size (Table 3). Multiple comparison of clinical stages revealed that there was no obvious difference in the positivity of Nampt expression. In addition, Nampt expression was not correlated with the depth of wall invasion. Furthermore, we found that the Nampt protein was not associated with lymph node metastasis $(P>0.05)$. Overall, these data indicate that there is no correlation of Nampt expression with clinicopathological features of CRC patients, such as gender, age, location, tumor size, clinical stages, depth of wall invasion, and lymph node metastasis.

\section{Effect of Nampt on colorectal cancer cell via- bility}

In order to study the effect of Nampt on CRC cell viability, we treated cells with different doses of recombinant human Nampt for 24, 48 and 72 h. Cell viability was analyzed using the CCK- 8 assay and calculated as indicated above. We found that Nampt treatment resulted in a significant increase of cell viability in HT29, Caco2, and HCT116 cells and peaked at $24 \mathrm{~h}$ after $100 \mathrm{ng} / \mathrm{ml}$ administration $(P<0.05)$ (Fig. $2 \mathrm{~A}-2 \mathrm{C}$ ) compared with it at $0 \mathrm{~h}$. These increases were reduced after 48 and $72 \mathrm{~h}$ in all three cell lines. No significant difference was observed in cells treated with low doses ( 25 and $50 \mathrm{ng} / \mathrm{ml}$ ). 

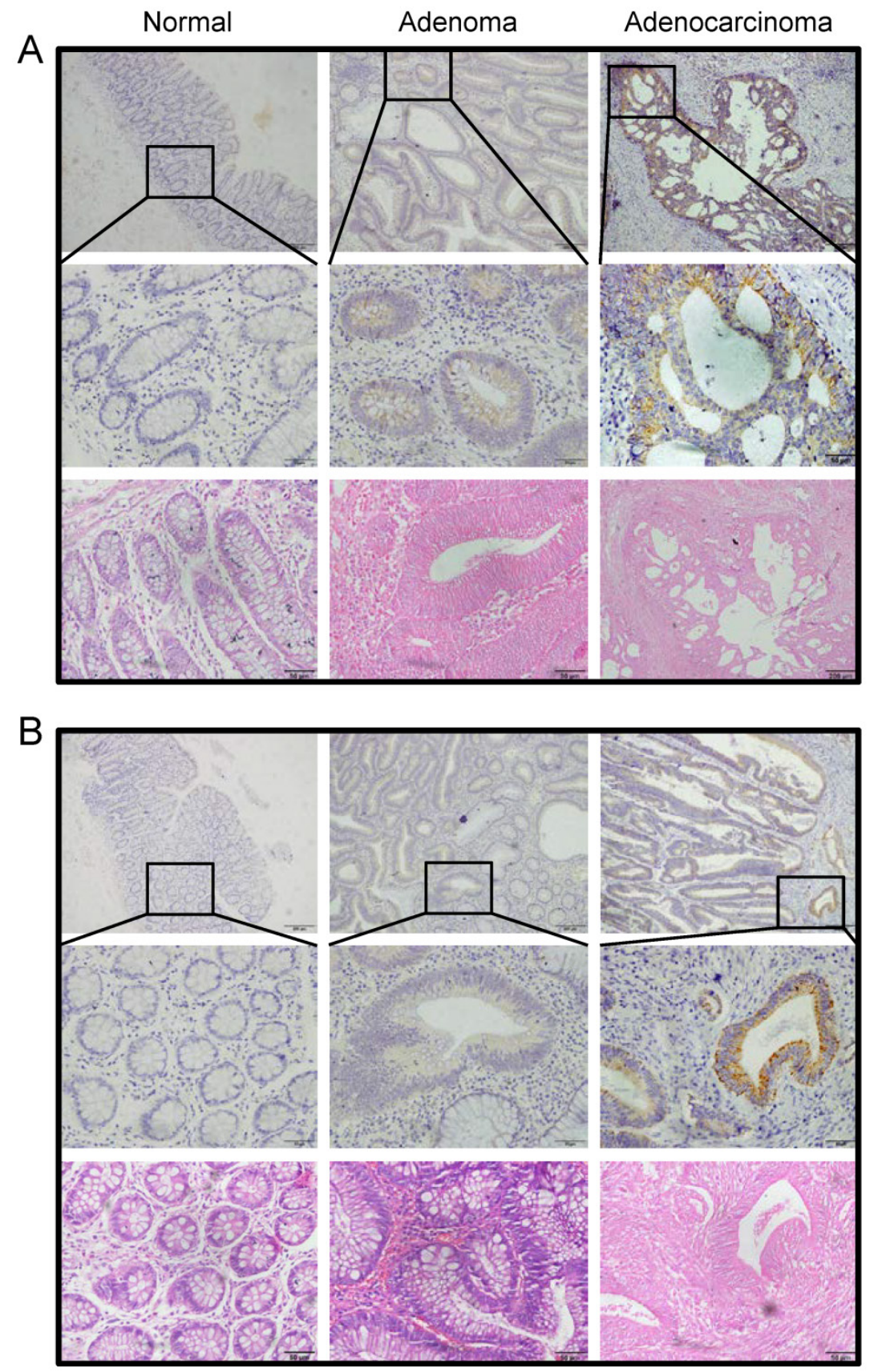

C

$\mathrm{D}$
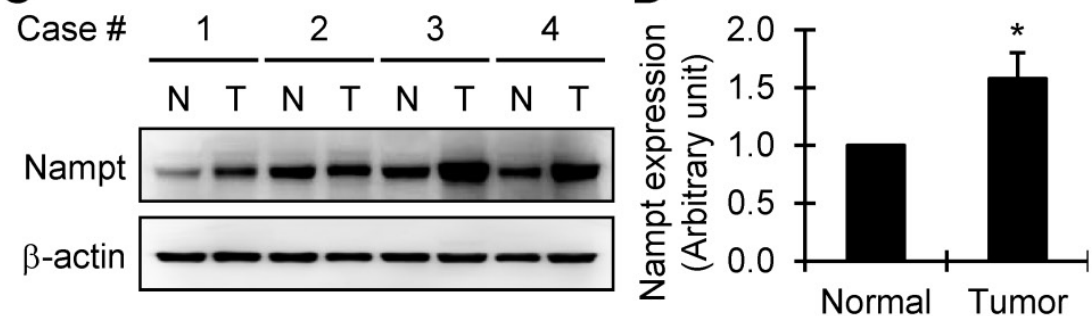

Fig. 1 Detection of Nampt expression in the colorectal tissues by immunohistochemical staining. Representative images of Nampt expression in adjacent normal, adenoma, and adenocarcinoma tissues of colon (A) and rectum (B), respectively, are shown (top panel, original amplification $\times 100$, scale bar 100 $\mu \mathrm{m}$; middle panel, original amplification $\times 400$, scale bar $50 \mu \mathrm{m}$; bottom panel, hematoxylin and eosin staining, original amplification $\times 400$, scale bar $50 \mu \mathrm{m}$ ). A brown color in the cytoplasm is considered as a positive staining. The overexpression of Nampt in tumor tissues was further confirmed by Western blot (C). Nampt expression was increased in tumor tissues (T) compared with matched adjacent normal colorectal tissues $(\mathrm{N})$ in four randomly selected patients and semiquantitatively analyzed after densitometry on the gels $(D)$. Bar represents mean \pm SEM. *, $P<0.05$. 

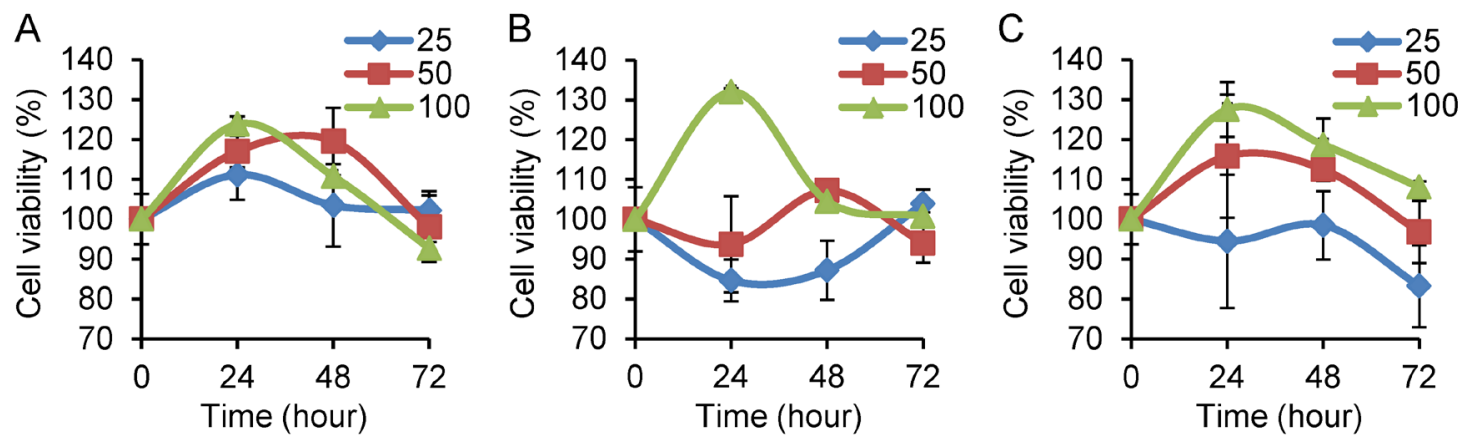

Fig.2 Determination of cell viability in colorectal cancer cell lines by CCK-8 assay. Cell viability was calculated as in percentage. HT29 (A), Caco2 (B), and HCT116 (C) cells were treated with Nampt at different doses for $0,24,48$, and $72 \mathrm{~h}$, respectively. The cell viability was represented as (average OD of treated groups at $0,24,48$ or $72 \mathrm{~h} /$ average $O D$ of untreated groups at the same time point) $\times 100$. A significant effect was observed in the three cell lines treated with Nampt at the concentration of $100 \mathrm{ng} / \mathrm{ml}$ for $24 \mathrm{~h}$.

Table 3 Nampt expression associated with the clinicopathological features of patients with colorectal carcinoma detected by immunohistochemistry

\begin{tabular}{|c|c|c|c|c|}
\hline \multirow{2}{*}{$\begin{array}{l}\text { Clinicopathological } \\
\text { feature }\end{array}$} & \multirow[t]{2}{*}{$\mathrm{n}$} & \multicolumn{2}{|c|}{ Nampt expression } & \multirow[t]{2}{*}{ P-value } \\
\hline & & Positive n (\%) & Negative $\mathrm{n}(\%)$ & \\
\hline \multicolumn{5}{|l|}{ Gender } \\
\hline Male & 28 & $16(57.1)$ & $12(42.9)$ & $0.516^{a}$ \\
\hline Female & 29 & $19(65.5)$ & $10(34.5)$ & \\
\hline \multicolumn{5}{|l|}{ Age } \\
\hline$<60$ & 17 & $9(54.9)$ & $8(47.1)$ & $0.392^{a}$ \\
\hline$\geq 60$ & 40 & $26(65.0)$ & $14(35.0)$ & \\
\hline Location & & & & $0.111^{a}$ \\
\hline Colon & 34 & $18(52.9)$ & $16(47.1)$ & \\
\hline Rectum & 23 & $17(73.9)$ & $6(26.1)$ & \\
\hline Tumor size & & & & $0.533^{a}$ \\
\hline$<5 \mathrm{~cm}$ & 34 & $22(64.7)$ & $12(35.3)$ & \\
\hline$\geq 5 \mathrm{~cm}$ & 23 & $13(56.5)$ & $10(43.5)$ & \\
\hline $\begin{array}{l}\text { Depth of wall inva- } \\
\text { sion }\end{array}$ & & & & $0.249 \mathrm{~b}$ \\
\hline Mucosa, submucosa & 5 & $4(80.0)$ & $1(20.0)$ & \\
\hline Muscularis propria & 10 & $8(80.0)$ & $2(20.0)$ & \\
\hline Serosa, subserosa & 42 & $23(54.8)$ & $19(45.2)$ & \\
\hline LN metastasis & & & & $0.946^{a}$ \\
\hline Yes & 23 & $14(60.9)$ & $9(39.1)$ & \\
\hline No & 34 & $21(61.8)$ & $13(38.2)$ & \\
\hline Clinical stage & & & & $0.173^{\mathrm{b}}$ \\
\hline I & 10 & $9(90.0)$ & $1(10.0)$ & \\
\hline II & 19 & $10(52.6)$ & $9(47.4)$ & \\
\hline III & 24 & $13(54.2)$ & $11(45.8)$ & \\
\hline IV & 4 & $3(75.0)$ & $1(25.0)$ & \\
\hline
\end{tabular}

\section{Inhibition of cell viability by Nampt-siRNA}

To a further series of experiments, we examined if knocking-down of Nampt via Nampt-siRNA results in a reduction of cell viability in CRC HT29, Caco2, and HCT116 cells. The cells were transfected with Nampt-siRNA using Lipofectamine RNAiMAX. Western blot assays confirmed that treatment with Nampt-siRNA resulted in a downregulation of Nampt protein expression in HT29, Caco2, and
HCT116 (Fig. 3A and 3B). Next, the effect of Nampt-siRNA on the cell viability for cultivated HT29, Caco2, and HCT116 cells was tested. No difference of cell viability was found between non-specific control siRNA and Nampt-siRNA groups at $48 \mathrm{~h}$ post-transfection (Fig. 3C). However as shown in Fig. 3D, Nampt-siRNA significantly inhibited cell viability of HCT116 and Caco2 cells, but not HT29 cell, as compared with cells transfected with non-specific control siRNA (NC) for $72 \mathrm{~h}$. This result suggested that Nampt knockdown potentially inhibits the HCT116 and Caco2 cell growth.

\section{Effect of Nampt inhibitor on colorectal cancer cell growth}

To further confirm the inhibition of Nampt on the cell viability of CRC cells, the Nampt inhibitor FK866 was applied. Cell growth was not affected by FK866 at $24 \mathrm{~h}$. Cell viability of the CRC cell lines HT29, Caco2, and HCT116 was decreased after FK866 administration for $72 \mathrm{~h}$ (Fig. 4A-4C). We also found a decrease of the cell viability of Caco2 at $48 \mathrm{~h}$ after treatment. Significant inhibition of cell growth was observed as a consequence of the treatment with FK866 at a concentration of $100 \mathrm{nM}$.

\section{Discussion}

In the present study we demonstrated that Nampt protein was indeed expressed in colorectal adenoma and overexpressed in colorectal carcinoma, compared with the adjacent normal colorectal tissue in which Nampt expression was negative. These results indicate that Nampt may play at least in part a role in the carcinogenesis and suggest the functionality of Nampt as a good progression marker for colorectal adenoma and carcinoma. 

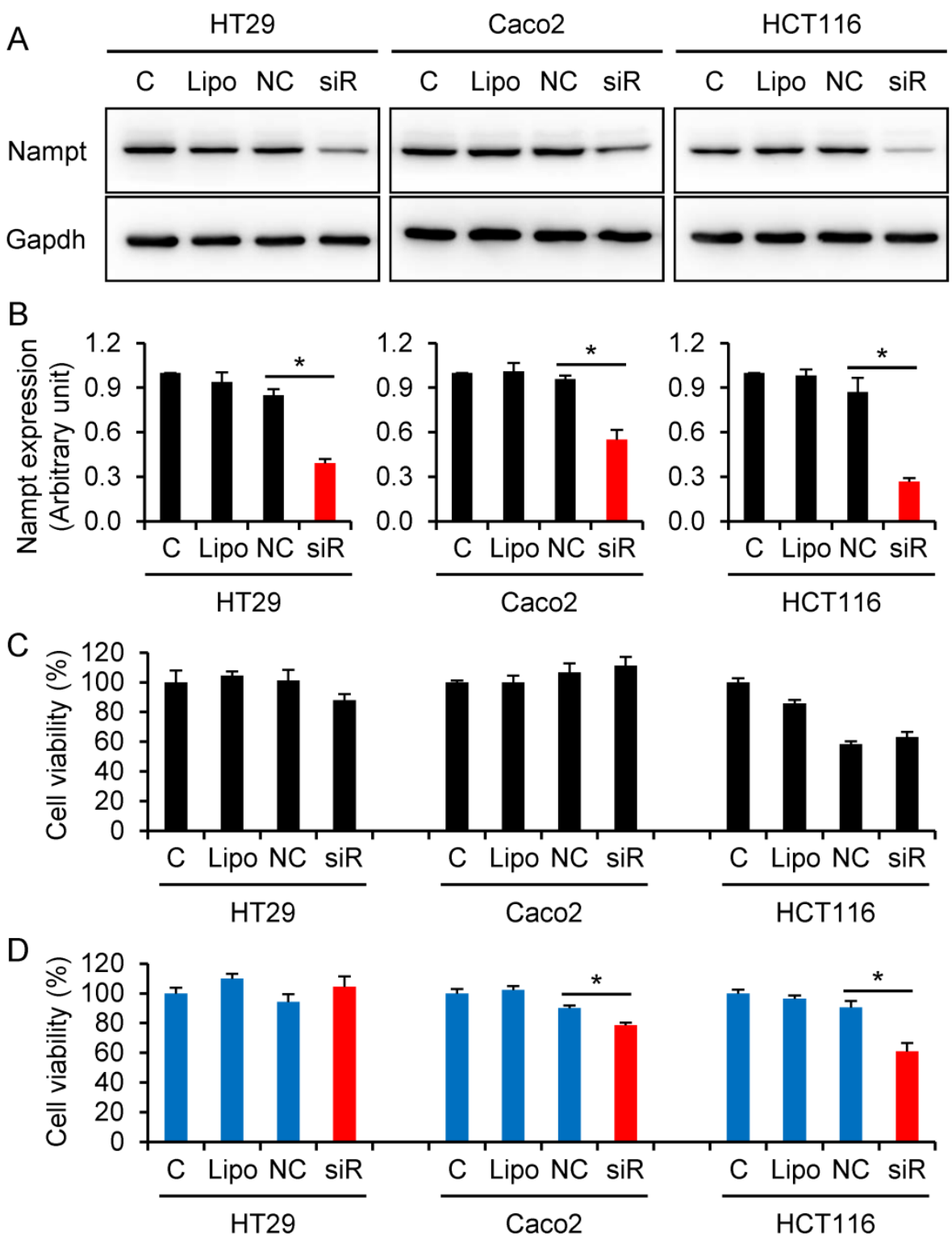

Fig.3 Decrease of colorectal cancer cell viability via knocking-down Nampt. (A) HT29, Caco2, and HCT1 16 cells were transfected with siRNA for $48 \mathrm{~h}$ and Nampt protein was detected by Western blotting. (B) Densitometry of signals in Western blot. (C) Cell viability (\%) was detected by CCK-8 assay at $48 \mathrm{~h}$ post-transfection. (D) Cell viability (\%) was detected by CCK-8 assay at $72 \mathrm{~h}$ post-transfection. $C$ control cells without transfection, Lipo control cells treated with transfection reagent Lipofectamine RNAiMAX alone, NC cells transfected with negative control of oligonucleotides, siR cells transfected with Nampt-siRNA. *, $P<0.01$ (siR vs. NC).

The overexpression of Nampt gene and protein was previously demonstrated in CRC using suppression subtractive hybridization (SSH) and phage display-derived antibodies, respectively $[15,16]$. In a recent study using a global mass spectrometry-based metabolomic approach, two tumor cell lines of different origins, namely the ovarian cancer cell line A2780 and the colorectal cancer cell line HCT116, were treated with FK866 in the presence and absence of nicotinic acid, resulting that the amino acids metabolism as well as purine and pyrimidine metabolism undergo significant changes in addition to the metabolic alterations in the glycolysis, the citric acid cycle, and the pentose phosphate pathway [27]. Their results gave some explanations about the chemical regulatory effect of the inhibitory and the metabolic role of Nampt during cancer disease development. Also in another study, a link between the genetic pathways (in that case the BRCA1) and the NAD-dependently metabolic pathways are cooperatively regulating the malignant progression of ovarian cancer since the knockdown or overexpression of BRCA1 was an effective way to induce an increase or decrease of Nampt-related NAD synthesis, because Nampt knockdown-mediated reduction in NAD levels was effective at inhibiting BRCA1 expression 
while the overexpression of Nampt led increased NAD levels and a subsequent elevation in BRCA1 levels in primary ovarian cancer cells and the other ovarian cell lines examined in the study [28]. Further, in another approach studying comparatively human breast cancer both in vivo and in vitro, high Nampt level was associated with aggressive pathological and molecular features, such as estrogen receptor negativity as well as HER2-enriched phenotypes [29].
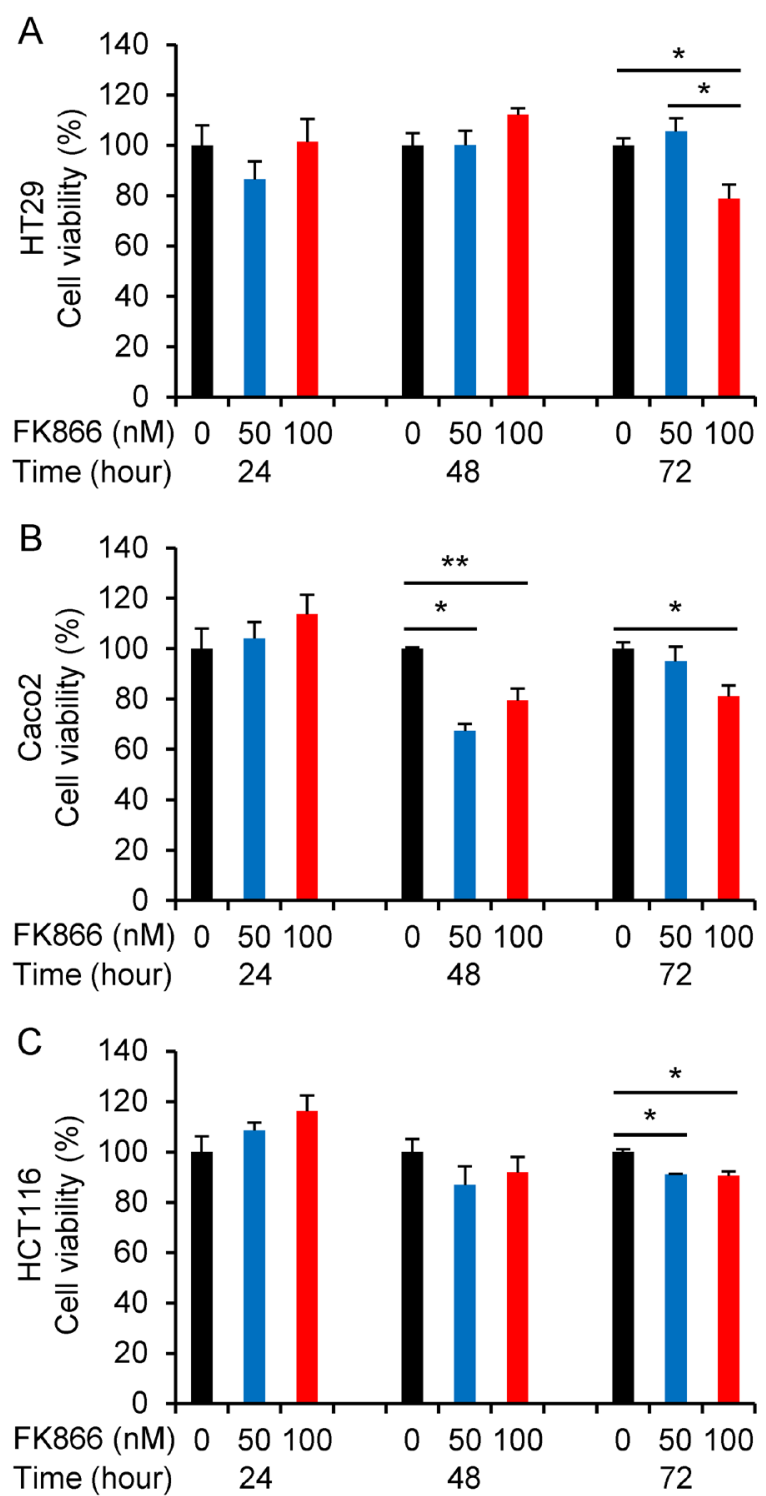

Fig.4 Regulative effect of the Nampt inhibitor FK866 on the cell viability. HT29 (A), Caco2 (B), and HCT116 (C) cells were treated without or with FK866 at different doses for 24,48 , and 72 hours, respectively. *, $\mathrm{P}<0.01$; **, $\mathrm{P}<0.05$ (treated vs. non-treated).

To our knowledge, the present study is the first report to evaluate the correlation between tissue Nampt and the clinicopathological characteristics of the colorectal malignant tumor. Here we found that Nampt expression in colorectal tissue was not corre- lated with gender, age, tumor size, depth of wall invasion, and lymph node metastasis. Besides, the expression of Nampt was not significantly different between the colon and rectum carcinomas. Moreover, in this study we found that the expression of Nampt in CRC tissue was not dependent on the clinical stages, although stage progression significantly correlated with serum Nampt levels has been found [30]. This is most likely due to the fact that our series of analyses were consisted of relatively small numbers of patients. This fact affects the statistical power of the examined series results. Thus, continued evaluation with the large numbers of patients may be needed to fully understand clearly the role of Nampt in CRC development and its relationship to clinicopathological features.

Although we have not measured the serum Nampt concentration in the current study, several previous reports from other groups have showed Nampt level in CRC patients were higher than those of controls. For example, Nampt levels in blood were significantly increased in CRC patients compared with normal controls [30]. A recent study also showed that the plasma Nampt levels in Chinese patients with early and advanced cancer were higher than those of normal controls [23]. However, the correlation between circulating Nampt level and clinical stage in colorectal cancer remains controversial. The serum Nampt level in the malign group (colon carcinoma) was not significantly different from that in the benign group [31]. Another recent study showed that there were no significant correlations between circulating Nampt level and the TNM staging for CRC, although patients with CRC had significantly higher level of serum Nampt than the control group both before and after adjustment for covariates (e.g. age and BMI) [24]. These data indicate that Nampt may represent good biomarker of colorectal malignant potential rather than staging. On the other hand, higher circulating level of Nampt may be an independent prognostic marker in some malignancies, such as non-muscle-invasive bladder cancer [32], endometrial cancer [20], malignant astrocytoma/glioblastoma [22], breast cancer [33], and gastric cancer [34]. However, the association of serum Nampt with prognosis in colorectal cancer has not been investigated and thus further study is needed in future.

It has been shown that the treatment of Nampt resulted in an increase of cell proliferation in breast cancer and prostate cancer cells $[35,36]$, whereas the Nampt inhibitor FK866 induces apoptosis in human liver carcinoma cells [25], hematologic malignant cells $[37,38]$, and gastric cancer cells [13]. Consistent with these previous studies in other cancer cells, the present study demonstrated that Nampt promoted CRC 
cell viability, whereas Nampt inhibitor FK866 suppressed the cell growth. This result was further confirmed by the knockdown of Nampt via siRNA. All these observations indicated that Nampt increases cell growth and may play an important role in the processes of carcinogenesis.

It has been reported that Nampt increases cell proliferation in prostate cancer and breast cancer cells by activating the phosphatidylinositol 3-kinase/Akt (PI3K-Akt) and mitogen activated protein kinase-extracellular signal regulated kinase $1 / 2$ and p38 (MAPK-ERK1/2 and p38) signaling pathways [35, 39], leading to cancer progression. The same conclusion may exist for the colorectal carcinoma cells. Inactivation of this pathway via the Nampt inhibitor FK866 may block the stimulatory effect due to the blockage of the involved downstream signal transduction pathways.

In conclusion, the present study demonstrated that Nampt was overexpressed in human colorectal adenoma and carcinoma. The immunoreactive staining of Nampt was negative in the adjacent normal colorectal tissue, weak in colorectal adenoma, and strong in colorectal carcinoma, which may represent tumor progression. Additionally, we demonstrated that recombinant human Nampt promoted, whereas Nampt inhibitor FK866 as well as Nampt-siRNA inhibited, cell viability in CRC cells. The involvement of Nampt in cell growth indicates that Nampt may play an important role in colorectal tumorigenesis. As a consequence, our results suggest that Nampt may be considered as a progression marker of colorectal tumor and a potential therapeutic target for the treatment of colorectal cancer.

\section{Abbreviations}

CCK-8: Cell Counting Kit-8; CRC: colorectal cancer; NAD: nicotinamide adenine dinucleotide; Nampt: nicotinamide phosphoribosyltransferase; PBEF: pre-B cell enhancing factor; SI: staining index

\section{Acknowledgements}

We thank Xuanguang Ye from the Department of Pathology at Jinshan Hospital for pathology assistance. This work was supported by grants from the Jinshan District Health Bureau (\#JSKJ-KTMS-201203) to $\mathrm{XL}$, and National Natural Science Foundation of China (\#81272880), the Shanghai Committee of Science and Technology (\#124119b1300) and Shanghai Municipal Health Bureau (\#2012-186) to GX.

\section{Authors' contributions}

$X \mathrm{Lv}$ contributed to the development of project and the performance of experiments. L Zhang performed the data analysis and prepared the figures. $Y$
Zhu collected the clinical samples and confirmed the diagnosis. HM Said participated in the data analysis and wrote the manuscript. J Shi performed immunohistochemistry. G Xu contributed to the design of experiment, the data analysis and the generation of figures and the writing of the manuscript. All authors read and approved the final manuscript.

\section{Competing Interests}

The authors have declared that no competing interest exists.

\section{References}

1. Siegel R, Ma J, Zou Z, Jemal A. Cancer statistics, 2014. CA Cancer J Clin. 2014; 64: 9-29.

2. Bosman FT, Hamilton SR, Lambert R. Colorectal cancer. In: Stewart BW, Wild CP, editors. World Cancer Report 2014. Lyon: International Agency for Research on Cancer; 2014: 558-75.

3. Koyanagi K, Bilchik AJ, Saha S, Turner RR, Wiese D, McCarter M, et al. Prognostic relevance of occult nodal micrometastases and circulating tumor cells in colorectal cancer in a prospective multicenter trial. Clin Cancer Res. 2008; 14: 7391-6.

4. Harriss DJ, Atkinson G, George K, Cable NT, Reilly T, Haboubi N, et al. Lifestyle factors and colorectal cancer risk (1): systematic review and meta-analysis of associations with body mass index. Colorectal disease : the official journal of the Association of Coloproctology of Great Britain and Ireland. 2009; 11: 547-63.

5. Giovannucci E, Michaud D. The role of obesity and related metabolic disturbances in cancers of the colon, prostate, and pancreas. Gastroenterology. 2007; 132: 2208-25.

6. Cui YM, Jiang D, Zhang SH, Wu P, Ye YP, Chen CM, et al. FOXC2 promotes colorectal cancer proliferation through inhibition of FOXO3a and activation of MAPK and AKT signaling pathways. Cancer letters. 2014; 353: 87-94.

7. Samal B, Sun Y, Stearns G, Xie C, Suggs S, McNiece I. Cloning and characterization of the cDNA encoding a novel human pre-B-cell colony-enhancing factor. Molecular and cellular biology. 1994; 14: 1431-7.

8. Fukuhara A, Matsuda M, Nishizawa M, Segawa K, Tanaka M, Kishimoto K, et al. Visfatin: a protein secreted by visceral fat that mimics the effects of insulin. Science. 2005; 307: 426-30.

9. Imai S. Nicotinamide phosphoribosyltransferase (Nampt): a link between NAD biology, metabolism, and diseases. Current pharmaceutical design. 2009; 15: $20-8$.

10. Revollo JR, Grimm AA, Imai S. The NAD biosynthesis pathway mediated by nicotinamide phosphoribosyltransferase regulates Sir2 activity in mammalian cells. J Biol Chem. 2004; 279: 50754-63.

11. Olesen UH, Thougaard AV, Jensen PB, Sehested M. A preclinical study on the rescue of normal tissue by nicotinic acid in high-dose treatment with APO866, a specific nicotinamide phosphoribosyltransferase inhibitor. Mol Cancer Ther. 2010; 9: 1609-17.

12. Galli M, Van Gool F, Rongvaux A, Andris F, Leo O. The nicotinamide phosphoribosyltransferase: a molecular link between metabolism, inflammation, and cancer. Cancer Res. 2010; 70: 8-11.

13. Bi TQ, Che XM, Liao XH, Zhang DJ, Long HL, Li HJ, et al. Overexpression of Nampt in gastric cancer and chemopotentiating effects of the Nampt inhibitor FK866 in combination with fluorouracil. Oncol Rep. 2011; 26: 1251-7.

14. Wang B, Hasan MK, Alvarado E, Yuan H, Wu H, Chen WY. NAMPT overexpression in prostate cancer and its contribution to tumor cell survival and stress response. Oncogene. 2011; 30: 907-21.

15. Hufton SE, Moerkerk PT, Brandwijk R, de Bruine AP, Arends JW, Hoogenboom HR. A profile of differentially expressed genes in primary colorectal cancer using suppression subtractive hybridization. FEBS letters. 1999; 463: 77-82

16. Van Beijnum JR, Moerkerk PT, Gerbers AJ, De Bruine AP, Arends JW, Hoogenboom HR, et al. Target validation for genomics using peptide-specific phage antibodies: a study of five gene products overexpressed in colorectal cancer. Int J Cancer. 2002; 101: 118-27.

17. Shackelford RE, Bui MM, Coppola D, Hakam A. Over-expression of nicotinamide phosphoribosyltransferase in ovarian cancers. International journal of clinical and experimental pathology. 2010; 3: 522-7.

18. Folgueira MA, Carraro DM, Brentani H, Patrao DF, Barbosa EM, Netto MM, et al. Gene expression profile associated with response to doxorubicin-based therapy in breast cancer. Clin Cancer Res. 2005; 11: 7434-43.

19. Shackelford R, Hirsh S, Henry K, Abdel-Mageed A, Kandil E, Coppola D. Nicotinamide phosphoribosyltransferase and SIRT3 expression are increased in well-differentiated thyroid carcinomas. Anticancer Res. 2013; 33: 3047-52.

20. Tian W, Zhu Y, Wang Y, Teng F, Zhang H, Liu G, et al. Visfatin, a potential biomarker and prognostic factor for endometrial cancer. Gynecologic oncology. 2013; 129: 505-12. 
21. Maldi E, Travelli C, Caldarelli A, Agazzone N, Cintura S, Galli U, et al. Nicotinamide phosphoribosyltransferase (NAMPT) is over-expressed in melanoma lesions. Pigment cell \& melanoma research. 2013; 26: 144-6.

22. Reddy PS, Umesh S, Thota B, Tandon A, Pandey P, Hegde AS, et al. PBEF1/NAmPRTase/Visfatin: a potential malignant astrocytoma/glioblastoma serum marker with prognostic value. Cancer biology \& therapy. 2008; 7: 663-8.

23. Chen M, Wang Y, Li Y, Zhao L, Ye S, Wang S, et al. Association of plasma visfatin with risk of colorectal cancer: An observational study of Chinese patients. Asia-Pacific journal of clinical oncology. 2013.

24. Fazeli MS, Dashti H, Akbarzadeh S, Assadi M, Aminian A, Keramati MR, et al. Circulating levels of novel adipocytokines in patients with colorectal cancer. Cytokine. 2013; 62: 81-5.

25. Hasmann M, Schemainda I. FK866, a highly specific noncompetitive inhibitor of nicotinamide phosphoribosyltransferase, represents a novel mechanism for induction of tumor cell apoptosis. Cancer Res. 2003; 63: 7436-42.

26. Wang X, Gui L, Zhang Y, Zhang J, Shi J, Xu G. Cystatin B is a progression marker of human epithelial ovarian tumors mediated by the TGF-beta signaling pathway. Int J Oncol. 2014; 44: 1099-106.

27. Tolstikov V, Nikolayev A, Dong S, Zhao G, Kuo MS. Metabolomics Analysis of Metabolic Effects of Nicotinamide Phosphoribosyltransferase (NAMPT) Inhibition on Human Cancer Cells. PLoS One. 2014; 9: e114019.

28. Li D, Chen NN, Cao JM, Sun WP, Zhou YM, Li CY, et al. BRCA1 as a nicotinamide adenine dinucleotide (NAD)-dependent metabolic switch in ovarian cancer. Cell cycle. 2014; 13: 2564-71.

29. Soncini D, Caffa I, Zoppoli G, Cea M, Cagnetta A, Passalacqua M, et al. Nicotinamide Phosphoribosyltransferase Promotes Epithelial-to-Mesenchymal Transition as a Soluble Factor Independent of Its Enzymatic Activity. J Biol Chem. 2014; 289: 34189-204.

30. Nakajima TE, Yamada Y, Hamano T, Furuta K, Matsuda T, Fujita S, et al. Adipocytokines as new promising markers of colorectal tumors: adiponectin for colorectal adenoma, and resistin and visfatin for colorectal cancer. Cancer science. 2010; 101: 1286-91.

31. Kosova F, Coskun T, Kaya Y, Kara E, Ari Z. Adipocytokine levels of colon cancer patients before and after treatment. Bratislavske lekarske listy. 2013; 114: 394-7.

32. Zhang K, Zhou B, Zhang P, Zhang Z, Chen P, Pu Y, et al. Prognostic value of serum nicotinamide phosphoribosyltransferase in patients with bladder cancer. Croatian medical journal. 2014; 55: 507-13.

33. Li XY, Tang SH, Zhou XC, Ye YH, Xu XQ, Li RZ. Preoperative serum visfatin levels and prognosis of breast cancer among Chinese women. Peptides. 2014; 51: 86-90.

34. Lu GW, Wang QJ, Xia MM, Qian J. Elevated plasma visfatin levels correlate with poor prognosis of gastric cancer patients. Peptides. 2014; 58: 60-4.

35. Patel ST, Mistry T, Brown JE, Digby JE, Adya R, Desai KM, et al. A novel role for the adipokine visfatin/pre-B cell colony-enhancing factor 1 in prostate carcinogenesis. Peptides. 2010; 31: 51-7.

36. Kim JG, Kim EO, Jeong BR, Min YJ, Park JW, Kim ES, et al. Visfatin stimulates proliferation of MCF-7 human breast cancer cells. Molecules and cells. 2010; 30: $341-5$

37. Muruganandham M, Alfieri AA, Matei C, Chen Y, Sukenick G, Schemainda I, et al. Metabolic signatures associated with a NAD synthesis inhibitor-induced tumor apoptosis identified by $1 \mathrm{H}$-decoupled-31P magnetic resonance spectroscopy. Clin Cancer Res. 2005; 11: 3503-13.

38. Nahimana A, Attinger A, Aubry D, Greaney P, Ireson C, Thougaard AV, et al. The NAD biosynthesis inhibitor APO866 has potent antitumor activity against hematologic malignancies. Blood. 2009; 113: 3276-86.

39. Adya R, Tan BK, Punn A, Chen J, Randeva HS. Visfatin induces human endothelial VEGF and MMP-2/9 production via MAPK and PI3K/Akt signalling pathways: novel insights into visfatin-induced angiogenesis. Cardiovasc Res. 2008; 78: 356-65. 